\title{
TEMPO-Oxidized Sacchachitin Nanofibers (TOSCNFs) Combined with Platelet-Rich Plasma (PRP) for Management of Dry Eye Syndrome
}

This article was published in the following Dove Press journal: International Journal of Nanomedicine

\author{
Hong-Liang Lin' \\ Ting-Huan $\mathrm{Wu}^{2}$ \\ Hsiu-O Ho (iD ${ }^{2}$ \\ Fang-Ching Chao ${ }^{2}$ \\ Meng-Huang Wu (1D ${ }^{3,4}$ \\ Der-Zen Liu ${ }^{5}$ \\ Ling-Chun Chen ${ }^{6}$ \\ Ming-Thau Sheu $\mathbb{D D}^{2}$ \\ 'School of Pharmacy, College of \\ Pharmacy, Kaohsiung Medical University, \\ Kaohsiung, Taiwan, Republic of China; \\ ${ }^{2}$ School of Pharmacy, College of \\ Pharmacy, Taipei Medical University, \\ Taipei, Taiwan, Republic of China; \\ ${ }^{3}$ Department of Orthopedics, Taipei \\ Medical University Hospital, Taipei, \\ Taiwan, Republic of China; ${ }^{4}$ Department \\ of Orthopedics, College of Medicine, \\ Taipei Medical University, Taipei, Taiwan, \\ Republic of China; ${ }^{5}$ Graduate Institute of \\ Biomedical Materials and Tissue \\ Engineering, College of Biomedical \\ Engineering, Taipei Medical University, \\ Taipei, Taiwan, Republic of China; \\ ${ }^{6}$ Department of Biotechnology and \\ Pharmaceutical Technology, Yuanpei \\ University of Medical Technology, \\ Hsinchu, Taiwan, Republic of China
}

Correspondence: Ling-Chun Chen; Ming-Thau Sheu

Taipei Medical University, $250 \mathrm{Wu}$-Hsing Street, Taipei II03I, Taiwan, Republic of China

Tel +886-2-2736I66I ext. 6112

Fax +886-2-23771942

Email d8801004@tmu.edu.tw;

mingsheu@tmu.edu.tw
Introduction: In this study, the combination of TEMPO-oxidized sacchachitin nanofibers (TOSCNFs) with chitosan-activated platelet-rich plasma (cPRP) was evaluated for remedying dry eye syndrome (DES).

Methods: TOSCNFs, designated T050SC, were generated. T050SC combined with chitosan-activated ( $c$ PRP) was formulated as eye drops for application for severe DES. To evaluate the effects of CPRP and TOSCNFs on the repair of corneal injury, in vitro studies were conducted using Statens Seruminstitut rabbit corneal (SIRC) epithelial cells for cell proliferation and cell migration assays, and a severe DES animal model using rabbits was established with benzalkonium chloride (BAC) treatment for the evaluation.

Results: Results showed that the optimal eye formulation contained PRP activated by 350 $\mu \mathrm{g} / \mathrm{mL}$ of the low-molecular-weight chitosan group (L3) combined with $300 \mu \mathrm{g} / \mathrm{mL}$ TO50SC (L3+T050SC). In the WST-1 cell-proliferation assay, L3 and L3+TO50SC significantly increased Statens SIRC cell proliferation after $24 \mathrm{hrs}$ of incubation. In the SIRC cell migration assay, the L3+TO50SC group showed a wound-healing efficiency of $89 \%$ after 24-hr treatment. After 5 days of treatment, Schirmer's test results did not simulate the dry eye animal model. Typical cornea appearance and eye fluorescein staining results showed that the L3 group had the best effect on improving cornea haze and epithelial damage.

Conclusion: This study has determined that TOSCNFs effectively promoted the healing effect on severe cases of corneal damage, and also might enhance the clinical application and medical potential of PRP in ophthalmology.

Keywords: TEMPO-oxidation, sacchachitin, PRP, dry eye syndrome, nanofibers

\section{Introduction}

Sacchachitin (SC) is a water-insoluble dry residue from Ganoderma tsugae produced by digesting with $\mathrm{KOH}$ and then bleaching with $\mathrm{NaClO}^{1} \mathrm{SC}$ is composed of approximately $40 \%$ chitin and $60 \% \beta$-1,3-glucan. ${ }^{1}$ Several potential applications of these two polysaccharides have been discussed previously. Wound healing ${ }^{1-3}$ and eye corneal regeneration ${ }^{4}$ for SC have been studied and reported. A promising wound healing has been demonstrated for an SC film composed of 10 50- $\mu \mathrm{m}$ microfibers formed by filtration followed by dry heat. For eye corneal regeneration, enhancement of corneal epithelial wound healing was observed with application of nanogel formed from micronized sacchachitin ( $\mathrm{mSC}$ ). However, the mechanical micronization of ball milling was only capable to disintegrate neighboring sacchachitin into microfibrils since SC 
was toughly organized together via H-bonding among hydroxyl groups or tangibly intertwined by single-chain SC.

In a previous study, ${ }^{5}$ two kinds of SC nanofibers (SCNFs) designated as MDSCNFs and TOSCNFs, respectively, were produced by either mechanical disintegration or oxidization with various concentrations (mmole/g SC) of $\mathrm{NaClO}$. In a diabetic wound-healing study, both SCN5 (mechanically fragmented SCNFs with NanoLyzer $^{\circledR}$ at 20,000 psi for five cycles) and T050SC (TEMPO-oxidized SCNFs produced with 5.0 mmole $\mathrm{NaClO} / \mathrm{g}$ SC) embedded in 2-acrylamide-2-methylpropane sulfonate (AMPS)-based wound dressings were showed to accelerate diabetic wound healing to effectively the same as normal skin tissues. T050SC/H furthermore fostered the growth of sweat glands and hair follicles in the healed wound enlightening that the wound had healed as functional tissue. It was concluded that TEMPO-oxidized SCNFs showed appreciably wellimproved healing of epithelial tissues. Most crucially being considered to use as a scaffold, TOSCNFs can be suspended in water resulting in the formation of hydrogels that offered an optimal 3D construct for cell growth and differentiation. ${ }^{6}$ These might be potentially applicable as a gel form for treating corneal disorders of the ocular surface caused by dry eye syndrome (DES).

DES is a syndrome of the tear film triggered by disturbances in the composition and quantity of tears. It might be attributed to 1) inadequacy of the aqueous phase of the tear, as in Sjögren's syndrome or lacrimal gland disease; 2) an imperfection of the mucin layer instigated by a vitamin A deficiency, trachoma, diphtheric keratoconjunctivitis, mucocutaneous disorders, and certain topical medications; or 3) abnormalities of the lipid tear layer caused by blepharitis and rosacea. ${ }^{7}$ Recently, Avila et al reported that platelet-rich plasma (PRP) was recommended to be a beneficial approach for healing several disorders of the ocular surface, ${ }^{8}$ including corneal ulcers ${ }^{9}$ and persistent ocular epithelial defects. ${ }^{10}$ It was also exhibited that PRP has an antiapoptotic effect on corneal stromal cells, ${ }^{11}$ and autologous platelets were instilled to manage macular holes. ${ }^{12,13}$ Autologous platelets in the form of eye-drops or as a plasma gel have additionally been used to manage several eye ill conditions depending on the desired effect and the purpose of the clinical intervention. In this way, PRP has shown great usefulness in the management of several ocular diseases. ${ }^{7}$

Thus, it was thought that because they possess better epithelial healing and hydrogel formation ability, TEMPO- oxidized sacchachitin nanofibers (TOSCNFs) combined with PRP would be synergistically beneficial for enhancing tissue-regenerating effects. Therefore, the purpose of this study was to evaluate the use of eye drops composed of TOSCNFs and autologous PRP for treating DES. To evaluate the effects of PRP and TOSCNFs on the repair of corneal injuries, in vitro studies were conducted using Statens Seruminstitut rabbit corneal (SIRC) epithelial cells for cell proliferation and cell migration assays and a severe DES animal model using rabbits was established with benzalkonium chloride (BAC) treatment for the evaluation.

\section{Materials and Methods Materials}

Ganoderma tsugae dry residue (G) (Kang Jian, Nantou, Taiwan), sodium hydroxide pellets (Macron, Radnor, PA, USA), potassium hydroxide (Fluka, Mexico City, Mexico), calcium chloride (Nacalai Tesque, Kyoto, Japan), sodium chlorite (80\%, Alfa Aesar, Ward Hill, MA, USA), sodium hypochlorite solution (5\% available chlorine, J.T. Baker, Pittsburgh, PA, USA), ethanol (Sigma-Aldrich, St. Louis, MO, USA), hydrochloride acid (Fluka), 2,2,6,6-tetramethyl1-piperidinyloxy (TEMPO) (98\%, Alfa Aesar), potassium bromide for the IR spectrum (Fluka), citric acid anhydrous (Riedel-de Haën, Seelze, Germany), acetic acid (SigmaAldrich), glycerol (Merck, Billerica, MA, USA), sodium bromide crystals (J.T. Baker), low-molecular-weight chitosan (Sigma Aldrich), PEG1000 (Sigma-Aldrich), N-acetylL-cysteine bioXtra (Sigma-Aldrich), L-ascorbic acid 2-phosphate sesquimagnesium salt hydrate (Sigma-Aldrich), minimum essential media (MEM) (1x, Gibco, Waltham, MA, USA), minimum essential medium-alpha (MEM- $\alpha$ ) (1x, Gibco), fetal bovine serum (FBS) (Gibco, InvivoGen, Waltham, MA, USA), phosphate-buffered saline (PBS) (10x, Corning, Corning, NY, USA), WST-1 reagent (Roche, Mannheim, Germany), antibiotic antimycotic solution (100x) (Corning), plasmocin (Gibco, InvivoGen), DMSO (Corning), and thiazolyl blue tetrazolium bromide (MTT reagent) (Alfa Aesar) were used as received without further purification. All water used was purified with the Milli-Q plus water purification system (Millipore, Billerica, MA, USA).

\section{Methods}

\section{Fabrication of TEMPO-Oxidized SCNFs}

TEMPO-oxidized SCNFs were fabricated following a report by Chao et al. ${ }^{5}$ In brief, ganoderma dry residues $(50 \mathrm{~g})$ were processed at $85^{\circ} \mathrm{C}$ for $24 \mathrm{hrs}$ with $400 \mathrm{~mL} 1$ 
$\mathrm{N} \mathrm{NaOH}$ solution. After treatment, the fibrous residue was gathered and rinsed with deionized water to remove any remaining $\mathrm{NaOH} . \mathrm{H}_{2} \mathrm{O}_{2}$ at an one-third weight ratio to the residue was then used for bleaching. After decolorizing, the fibrous pulp was cleaned thoroughly with doubledistilled (dd) $\mathrm{H}_{2} \mathrm{O}$. The fibrous pulp, so-called SC, was assembled by filtration then lyophilized. For oxidation by TEMPO, $5 \mathrm{~g}$ of $\mathrm{SC}$ was suspended in $375 \mathrm{~mL} \mathrm{ddH_{2 }}$ $\mathrm{O}$ comprising $0.0625 \mathrm{~g}$ TEMPO and $0.625 \mathrm{~g}$ sodium bromide. The TEMPO-mediated oxidation of the SC slurry was started by adding $5 \% \mathrm{NaClO}$ (aq.) at an $\mathrm{NaClO}$ amount of $5.00 \mathrm{mmol} / \mathrm{g} \mathrm{SC}$ to the slurry mixture with continuous stirring at room temperature. During reaction, pH 10 was preserved by adding $5 \mathrm{~N} \mathrm{NaOH}$ and $1 \mathrm{~N} \mathrm{HCl}$ for $2 \mathrm{hrs}$ or until no alkali solution was consumed. Thereafter, oxidation was stopped by adding $5 \mathrm{~mL}$ ethanol followed by adding $200 \mathrm{~mL}$ acetone to precipitate the TEMPO-oxidized SC. The TEMPO-oxidized SC fibrous slurry was then dialyzed with OrDial ${ }^{\circledR}$ D80 (MWCO: 6000 8000) for 7 days in $\mathrm{ddH}_{2} \mathrm{O}$ at room temperature. The dialyzed slurry was further homogenized with highpressure homogenizer $\left(\right.$ NanoLyzer $\left.{ }^{\circledR} \mathrm{N}-2\right)$ under 20,000 psi for one cycle, and then lyophilized. SC oxidized with TEMPO followed the conditions as described above was designated as T050SC.

\section{Physical and Chemical Characterizations of TEMPO-Oxidized SCNFs}

Physical characteristics including fourier transform infrared (FT-IR) spectra, ${ }^{13} \mathrm{C}$ cross-polarized magic-angle spinning (CP-MAS) NMR spectra, X-ray diffraction (XRD) spectra, determination of the size and zeta Potential $(\zeta)$, evaluation of gel-forming properties, transmission electronic microscopy (TEM), scanning electronic microscopy (SEM), water-retention ability, were measured followed the same procedure as that reported by Chao et al. ${ }^{5}$ The carboxylate and aldehyde contents of T050SC were determined according to the same method as that reported by Chao et al. ${ }^{5}$

\section{Preparation of PRP from Rabbit Blood}

Blood $(9 \mathrm{~mL})$ was collected from the marginal ear vein of New Zealand white dutch rabbits. Whole blood was loaded into a blood-collecting tube coated with anticoagulant citrate dextrose solution A (ACD-A), then centrifuged at $2500 \mathrm{rpm}(720 \mathrm{~g})$ for 15 mins. After centrifugation, the blood was separated into three layers. We collected the middle layer which contained the buffy coats and the upper layer which contained the platelet-poor plasma for a second centrifugation. The second centrifugation was carried at $4800 \mathrm{rpm}(2650 \mathrm{~g})$ for $15 \mathrm{~min}$, and then the PRP was at the bottom of the tube. We diluted the PRP to a final concentration of $4.75 \times 10^{4}$ platelets $/ \mu \mathrm{L}$ for further application. Low-molecular-weight chitosan $(135 \mu \mathrm{g})$ was added to each milliliter of PRP for activation just before its usage.

\section{Evaluation of the Effects of PRP Combined with TEMPO-Oxidized SCNFs on Cell Proliferation and Cell Migration of SIRC Fibroblasts}

SIRC fibroblasts were used in the same culture conditions as described above. Six different treatments were used to evaluate proliferation including PRP, low-molecularweight chitosan-activated PRP (LPRP), $300 \mu \mathrm{g} / \mathrm{mL}$ T050SC combined with PRP, and $300 \mu \mathrm{g} / \mathrm{mL}$ T050SC combined with LPRP. Cell proliferation was determined at $72 \mathrm{hrs}$ with the WST-1 reagent. The WST-1 assay was the same as described above.

SIRC fibroblasts (purchased from BCRC, Bioresource Collection and Research Center) were used in the same culture condition as described above. Before adding treatment medium, cells were scraped out of a straight line with a $200-\mu \mathrm{L}$ pipette tip at the bottom of each well. Then, four different treatments were used to evaluate cell migration including PRP, $300 \mu \mathrm{g} / \mathrm{mL}$ T050SC, 5\% PRP, 5\% LPRP, and $300 \mu \mathrm{g} / \mathrm{mL}$ T050SC combined with $5 \%$ PRP. Photos were taken of cells under a microscope at $0,6,18$, and $24 \mathrm{hrs}$ to determine cell migration.

\section{Rabbit Model of Severe DES}

Captivation of all animals was conducted in keeping with a protocol (no. LAC-2017-0152, 2017-06-07) approved by the Laboratory Animal Center of Taipei Medical University, and all experiments were implemented in accordance with the Guidelines for the Care and Use of Laboratory Animals. Animals were accommodated under a 12-hr light-dark cycle with free admittance to water and food. The rabbit model of severe DES was modified from that reported by Tseng et al. ${ }^{14}$ DES was induced in New Zealand white Dutch rabbits aged 15 weeks by $20 \mu \mathrm{L} 0.1 \%$ benzalkonium chloride three times a day for 3 weeks, and then benzalkonium chloride was increased to $0.2 \%$ for a week. After the DES model was successfully induced, four different treatments including $1 \%$ PBS, LPRP, T050SC, and $300 \mu \mathrm{g} / \mathrm{mL}$ T050SC combined with LPRP were applied twice a day for 5 days.

To evaluate the healing outcomes, we used corneal fluorescein staining. Rabbits were anesthetized with 
1 1.5 mL Zoletil+Xylazine (Roumpun) $(5 \mathrm{mg} / \mathrm{kg}+5 \mathrm{mg} /$ $\mathrm{kg}$ ), and laid flat on an operating table. Corneal fluorescein staining was conducted using fluorescein sodium ophthalmic strips (Fluoro Touch, New Delhi, India). Strips were first wetted with PBS and placed on a rabbit's cornea. After staining, the corneal topography was evaluated by cobalt-blue light ophthalmoscopy (Topcon Medical Systems, Oakland, NJ, USA).

\section{Histological Analysis}

On day 5, treated rabbit corneas were sampled and fixed in a $10 \%(\mathrm{w} / \mathrm{v})$ formaldehyde in aqueous phosphate buffer solution. Collected samples were then dehydrated with a gradient series of ethanol and implanted within paraffin. Embedded tissue blocks were sectioned and stained with hematoxylin and eosin (H\&E). Histological images of all stained samples were filmed underneath of optical microscopy (BX41, Olympus, Tokyo, Japan).

\section{Statistical Analysis}

All physical and chemical measurements were presented as the mean \pm standard deviation (SD). All in vitro and in vivo studies are presented as the mean \pm standard error of the mean (SEM). Data were compared among treatment groups using one-way Analysis of Variance (ANOVA), and $p<0.05$ was regarded as statistical significance.

A

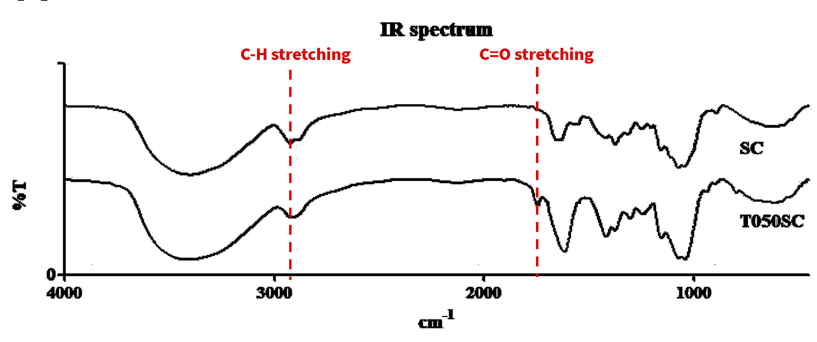

C

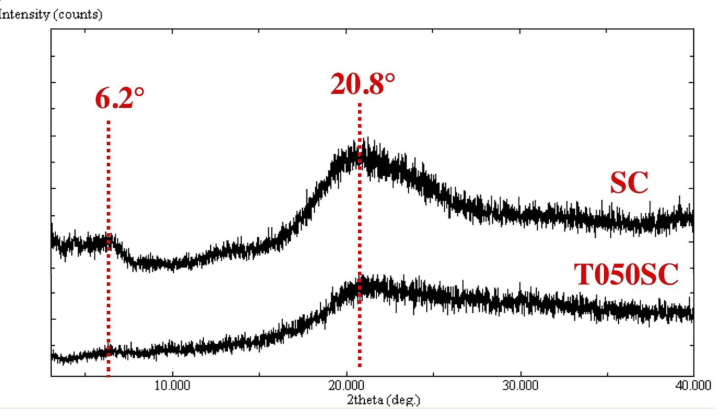

\section{Results}

\section{Physical and Chemical Characterization of TEMPO-Oxidized SCNFs}

SCNFs obtained by TEMPO oxidization with $5.0 \mathrm{mmole} / \mathrm{g}$ of SC were designated as T050SC. Several physical characteristics including FTIR, CP-MASS ${ }^{13} \mathrm{C}$ ssNMR, and $\mathrm{X}$-ray powder diffraction were measured and were reported in a previous publication. ${ }^{5}$ Those results are summarized as follows. Both FTIR spectra of T050SC and SCNF (Figure 1A) showed representative cellulosic or chitin peaks, ie, $\mathrm{O}-\mathrm{H}, \mathrm{C}-\mathrm{H}$, and $\mathrm{C}-\mathrm{O}$ stretching vibrations at 3400,2900 , and $1060 \mathrm{~cm}^{-1}$, respectively. After oxidation, T050SC appeared a sharp peak at $1720 \mathrm{~cm}^{-1}$ which was $\mathrm{C}=\mathrm{O}$ stretching, while the moderate $\mathrm{C}-\mathrm{H}$ stretching peak at $2920 \mathrm{~cm}^{-1}$ had decreased. Both peak changes were attributed to the transformation of $-\mathrm{CH}_{2} \mathrm{OH}$ to $-\mathrm{COOH}$ at $\mathrm{C} 6$ in the T050SC structure. Figure $1 \mathrm{~B}$ shows ${ }^{13} \mathrm{C}$ crosspolarized magic-angle spinning (CP-MAS) NMR spectra for T050SC. Since T050SC was mainly composed of $\beta$-1,3-glucan and chitin, it showed carbon signal for $\mathrm{C} 1$, $\mathrm{C} 2, \mathrm{C} 3, \mathrm{C} 4, \mathrm{C} 5$, and $\mathrm{C} 6$ located at 103, 62, 74, 87, 77, and $68 \mathrm{ppm}$, respectively. Basically, the carbonyl group of acetyl amide was presented in chitin structure showing a carbon peak for $\mathrm{C} 8$ centered at $23 \mathrm{ppm}$. Further, it was expectable that the signal intensity of the $\mathrm{C} 6$ peak should decrease accordingly with oxidation of the hydroxyl group

B

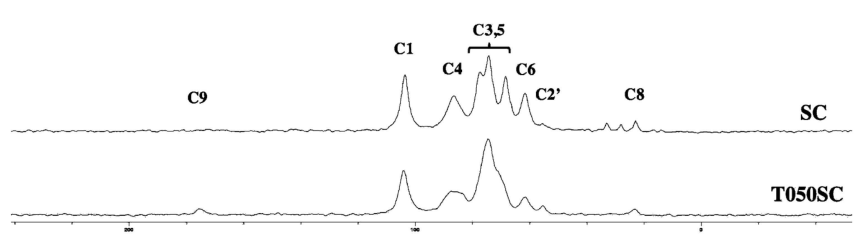

D

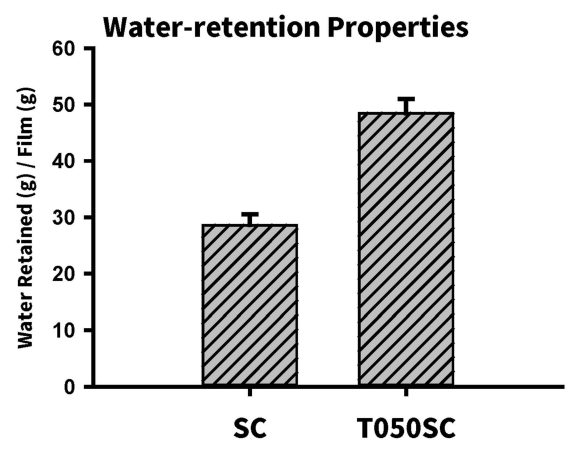

Figure I Physical characterization of mechanically nanosized sacchachitin (SC) and chemically TEMPO-oxidized sacchachitin nanofibers (T050SC). (A) FT-IR spectrum, (B) ${ }^{13} \mathrm{C}$ ssNMR, (C) X-ray powder diffraction, (D) Water retention. 
on C6. On the other hand, a new peak at 176 ppm was appeared resulting from transformation of primary alcoholic group $\left(-\mathrm{CH}_{2} \mathrm{OH}\right)$ into a carbonyl group $(-\mathrm{C}=\mathrm{O})$ by TEMPO oxidation. These alterations additionally confirmed the conversion of the C6 primary hydroxyl group to a carboxylate group. There was also a slight change in the C5 peak. C5 exhibited an upfield chemical shift from 77 to $74 \mathrm{ppm}$, which overlapped with the C3 peak. This upfield shift might have been triggered by the newly created carboxylate group based on observation from the 3D structure.

As illustrated in Figure 1C for the X-ray powder diffraction analysis, T050SC nanofibers possessed an amorphous form with a lower crystallinity as well. There appeared two main peaks at $2 \theta=6.2^{\circ}$ and $20.8^{\circ}$. The amorphous form might be due to the existence of T050SC fiber size in the nanoscale range, since as small as nanosize of the fiber lengths was expected to offer a more-amorphous structure. This amorphous structure also facilitated the resuspension of lyophilized fibers. Acid-base titration method was employed to determine the carboxylate and aldehyde contents in T050SC to be 1.803 and $0.119 \mathrm{mmol} / \mathrm{g}$, respectively. With the formation extent of aldehyde/g SC less than $1 \mathrm{mmol}$, it warranted that TEMPO oxidation had minor concerns of potential toxicity. The size and SD for T050SC nanofibers were observed to be 539.2 and $49.3 \mathrm{~nm}$, respectively, while its zeta potential was measured to be $-51.1 \mathrm{mV}$. This more negativity than that for SC nanofibers without TEMPO oxidation also definitely verified that the C6 primary hydroxyl group had been transformed into a carboxylate group. With the contribution by greater hydration abilities with the existence of hydrophilic carboxylate groups on the surface of these nanofibers, T050SC had waterretention abilities of $48.48 \pm 2.54 \mathrm{~g}$ water retention $/ \mathrm{g}$ film as shown in Figure 1D.

Figure $2 \mathrm{~A}$ shows that immediately after dispensing $2 \%$ $(\mathrm{w} / \mathrm{v})$ T050SC in water, T050SC was viewed like wet fibrous hydrogels. Thirty minutes after stirring, T050SC displayed a gel form monitored by the vial-inversion method. However, the gel-forming character of TS50SC vanished at the 60-min time point, and it never happened again thereafter. The results indicated that the transformation of C6 hydroxyl group into carboxylic group led to negatively charged nanofibril surfaces, which allowed the formation of homogeneous electrostatically stabilized aqueous colloidal suspensions. In TEM as shown in Figure 2B, T050SC had fiber lengths of $825.54 \pm 109.18$ $\mathrm{nm}$ and widths of $27.87 \pm 5.04 \mathrm{~nm}$. T050SC had lesstangled fibers due to its higher zeta potential of -51.6 $\mathrm{mV}$. With a higher negative charge on the surfaces of nanofibers, the nanofibers of T050SC displayed better separation and dispersion. In the SEM morphology as shown in Figure 2C, T050SC nanofibers existed as highly spongy structure, not only on the surface of the lyophilized sponge film but also on its cross-section.

\section{In vitro and in vivo Regeneration Studies for DES}

The T050SC dressing was used to evaluate chronic wound healing for DES based on previous chemical and physical evaluations. In the WST-1 assay of SIRC fibroblast cells, cell viability in the T050SC groups was first evaluated. T050SC was demonstrated to be highly biocompatible at all concentrations as shown in Figure 3A. However, the higher the concentration, the more viscous the solution was, and we also considered the transparency which are two important properties that should be evaluated in developing eye drop products. We picked $300 \mu \mathrm{g} / \mathrm{mL}$ for further experiments. T050SC was combined with PRP or L3 to assess cell proliferation, and results illustrated in Figure 3B show that the PRP and PRP+T050SC groups did not produce quite as promising cell-proliferation effects. This possibly indicates that T050SC had less effect of activating platelets to release growth factors to promote cell proliferation. Combining L3, which is activated-PRP with chitosan $(135 \mu \mathrm{g} / \mathrm{mL})$, with T050CS showed promising cell-proliferation effects. L3 and L3+T050SC showed over $150 \%$ cell viability after $24 \mathrm{hrs}$. However, L3 had the highest cell proliferative effect at around $180 \% \pm 3.75 \%$ cell viability. After $72 \mathrm{hrs}$ of incubation, L3 and L3+T050SC dropped to $134.86 \% \pm 12.87 \%$ and $142.16 \% \pm 7.86 \%$ cell viability, respectively. In comparison between 24 and $72 \mathrm{hrs}$, cell viabilities of L3 and L3 $+\mathrm{T} 050 \mathrm{SC}$ dropped $25 \%$ and $13 \%$, respectively. L3 showed a greater decrease indicating that T050SC can provide a more-viscous formulation for the sustained release of growth factors. In other words, TOSCNF formulations can work as plant-based scaffolds in this system and provide a sustained-release effect of growth factors released by activated PRP. Sustained release can prolong the effect of the formulation which can decrease the drug administration time for higher convenience and patient compliance. Since L3+T050SC showed the most 


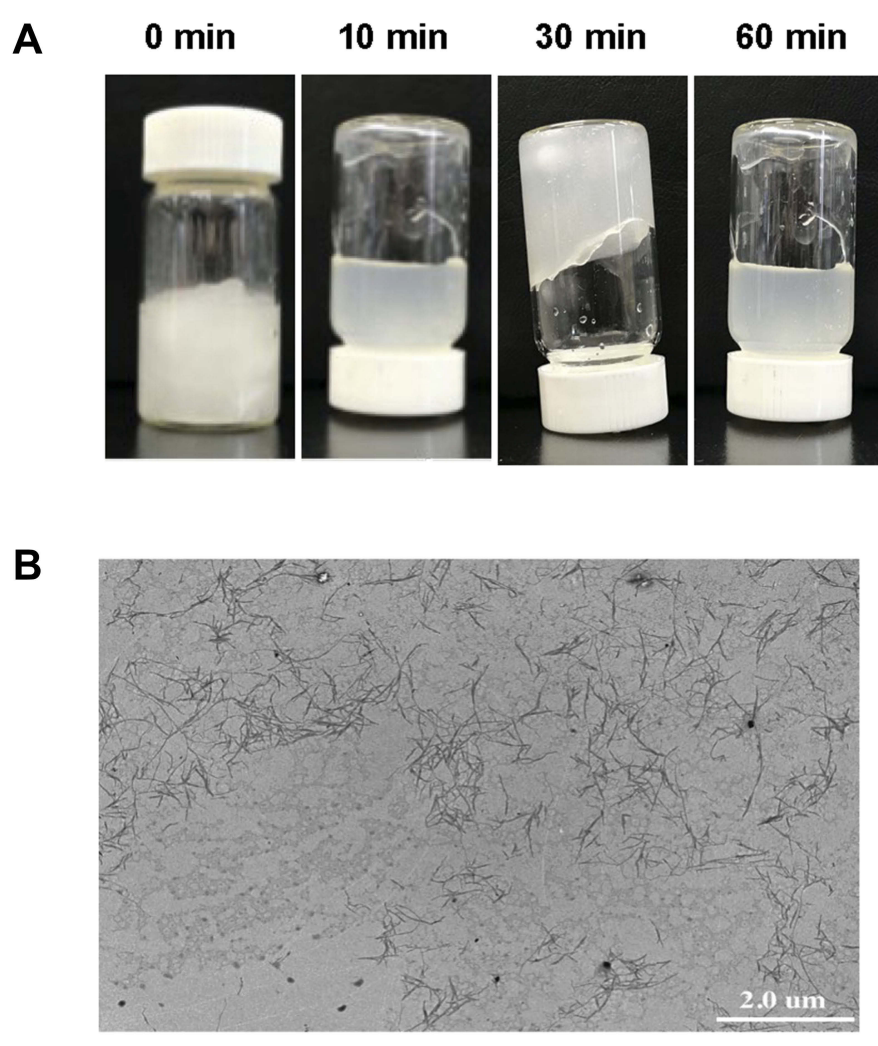

C

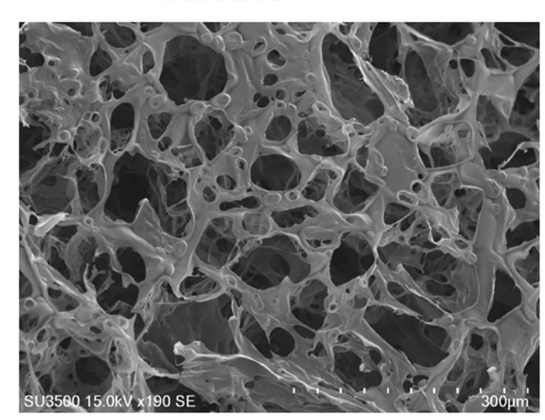

$2 \mathrm{hr}$

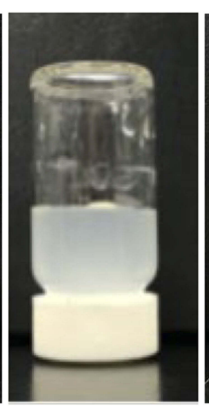

$6 \mathrm{hr}$

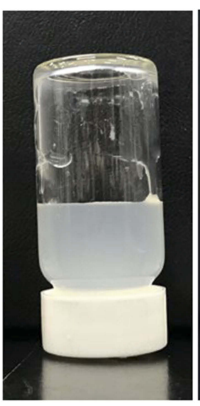

$24 \mathrm{hr}$

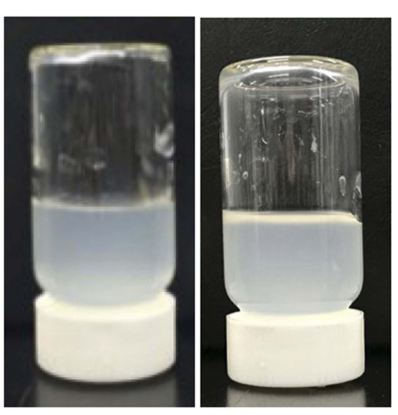

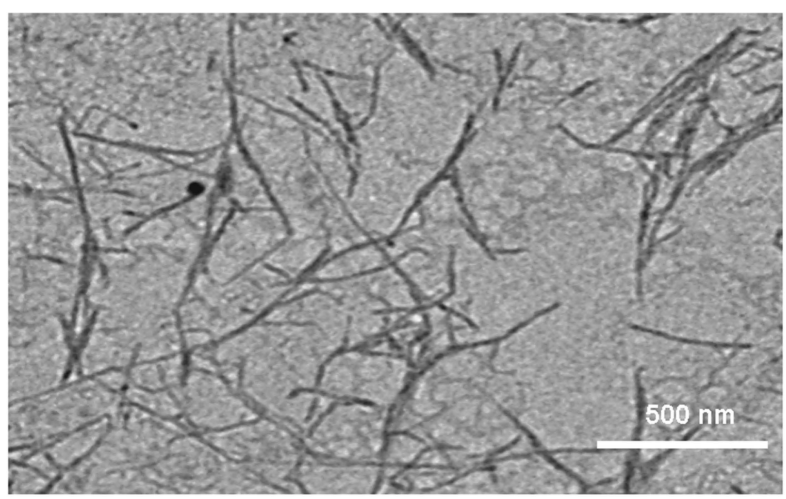

\section{Section}
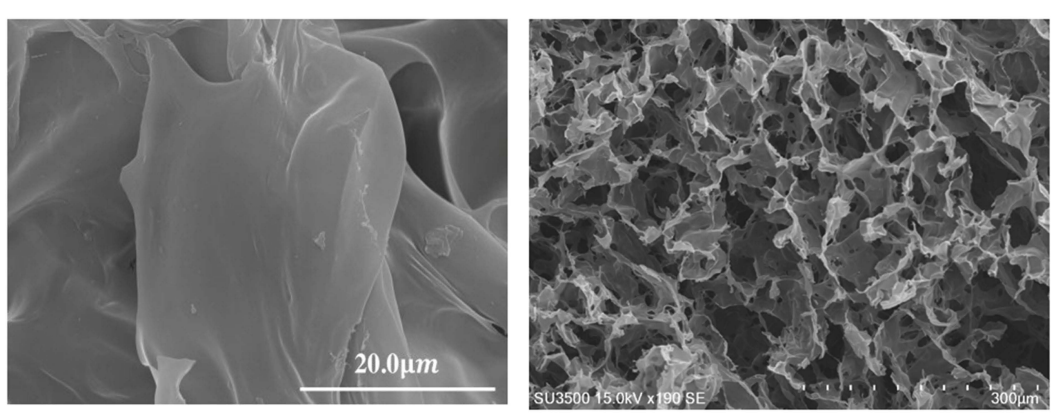

Figure 2 Evaluation of the gel-forming properties of TEMPO-oxidized sacchachitin by the vial-inversion method. (A) A T050SC sample (2\% w/v) was stirred at $700 \mathrm{rpm}$ at room temperature and examined at predetermined intervals. (B) TEM images of T050SC nanofibers at two magnifications (left panel: $10^{4} X$; right panel: $\left.2.5 \times 10^{4} X\right)$. (C) SEM images of $2 \%(w / v)$ lyophilized T050SC sponge film on the surface and the section area.

promising effect on cell proliferation, it was selected to perform subsequent experiments.

In the migration assay, we evaluated formulations which promoted SIRC cell migration for $24 \mathrm{hrs}$, and results of photo imaging of migration and quantification of gap closure are shown in Figure 3C and D, respectively. PRP, L3, and L3+T050SC all showed a significant difference in gap closure compared to the control group at the same time point. In contrast, T050SC alone did not show a significant difference with the control. In other words, T050SC alone had a limited effect on promoting cell migration. In previous studies, multiple growth factors from PRP were reported to promote cell migration. PRP, L3, and L3+T050SC all showed significant differences with the control group in the first $6 \mathrm{hrs}$, and these significant differences persisted until $24 \mathrm{hrs}$. In other words, growth factors in PRP can promote cell migration and showed a significant difference at $6 \mathrm{hrs}$. However, L3+T050SC showed the most outstanding cell migratory effect among all groups. Comparing L3 + T050SC to L3, one can see that L3+T050SC had a smaller cell gap at the late time point of $24 \mathrm{hrs}$, which may have resulted from T050SC providing sustained release of growth factors. L3+T050SC promoted cell 
A

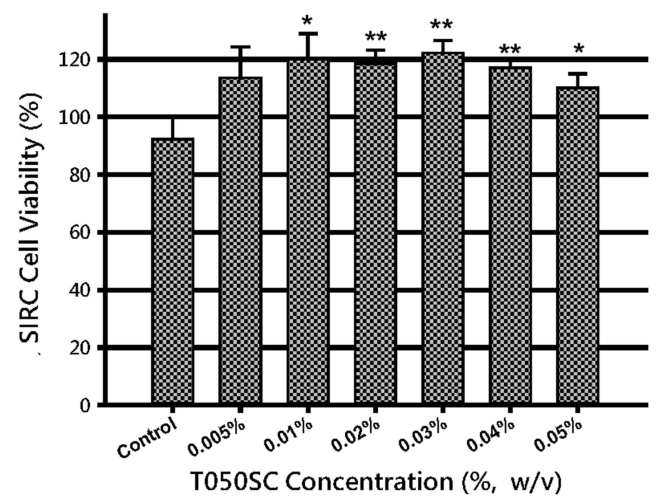

C

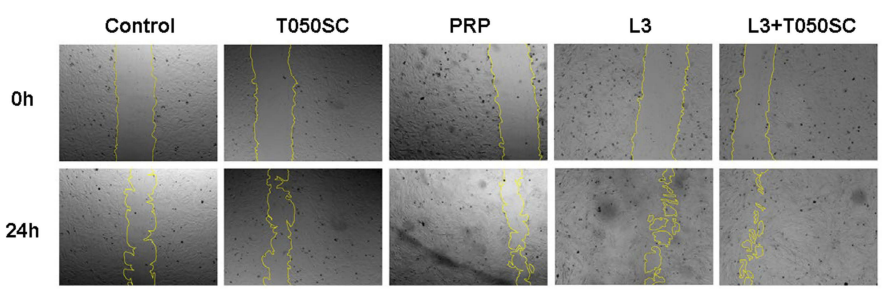

B

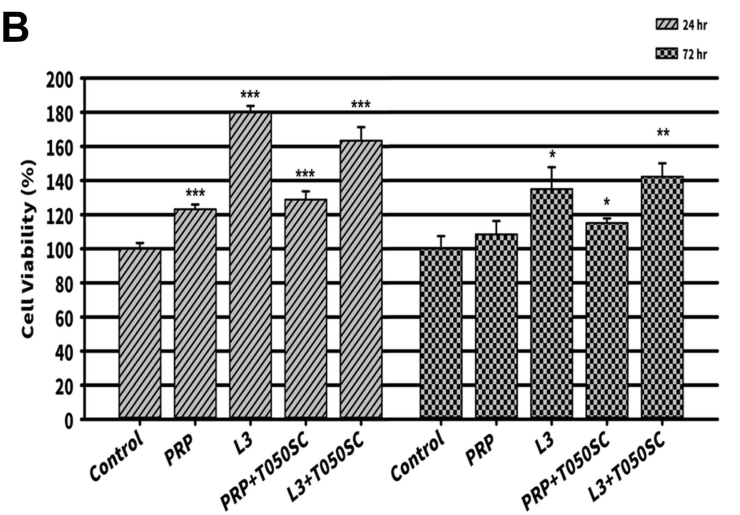

D

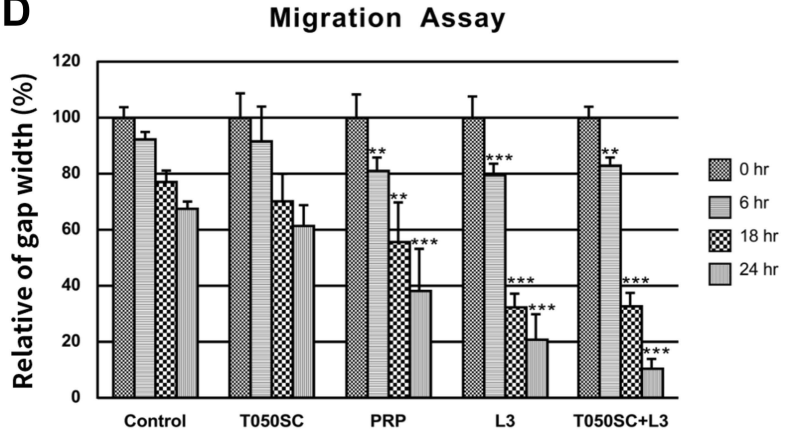

Figure 3 (A) WST-I assay of cell viability of SIRC fibroblasts treated with various concentrations of T050SC. Results are expressed as the mean \pm standard deviation; $n=5$. ${ }^{*} p<0.05,{ }^{*} p<0.001$ compared to the control (adding culture medium only). (B) WST-I assay of SIRC fibroblast cell proliferation of the combination of platelet-rich plasma (PRP, L3) and TEMPO-oxidized SCNFs (T050SC). Results are expressed as the mean \pm standard deviation; $n=5$. $* p<0.05$, $* * p<0.00$ I, $* * * p<0.000 \mathrm{I}$ compared to the control (adding culture medium only). (C) Cell migration assay of SIRC fibroblast cells (adding culture medium only as the control). Photos were collected at 0 and 24 hrs under phase-contrast microscopy. (D) Relative $\%$ of Gap Width was quantified by an image analysis. Results are expressed as the mean \pm standard deviation; $n=5$. $* *<<0.00$, $* * * p<0.0001$ compared to the control (adding culture medium only).

migration, and $89 \%$ of the wound had healed, which was the best result in this experiment.

In an in vivo study, we applied L3+T050SC to severe DES, which was generated in 15-week-old New Zealand white dutch rabbits by applying $0.1 \%$ BAC t.i.d. for 3 weeks and $0.2 \%$ BAC t.i.d. for 1 week. Figure $4 \mathrm{~A}$ shows that healthy rabbit eyes had a transparent cornea and no corneal fluorescein staining. After BAC induction, the cornea became cloudy, and one can also see fluorescein staining of the cornea. In severe DES, the cornea becomes cloudy, and the cornea can also be damaged, which will affect the visual ability of the patient. On day 0 of treatment, one can also see the cornea status of all groups with severe DES. After treatments with L3 and L3+T050SC, the cornea had again become transparent, and the corneal ulcer was also relieved. In contrast, in the control and T050SC groups, the cornea remained non-transparent, and the ulcer could still be observed. Corneal fluorescence staining as illustrated in Figure 4B showed no staining in the T050SC, L3, and L3+T050SC groups after 5 days of treatment. In other words, the corneal damage of these three groups had been relieved. Under cobalt-blue light ophthalmoscopy, one can see that the L3 group had the best optical properties compared to the others. However, the L3+T050SC groups showed a better outcome of corneal ulcer relief than L3.

Histological images of corneal severity are shown in Figure 4C, and qualification and quantification evaluations of the corneal severity level after 5 days of treatment are illustrated in Table 1. Results showed that there were about three to five epithelial layers properly aligned and well organized on the surface of the cornea, which showed a more-purple stain under H\&E staining in the healthy rabbit eye cornea. The central thickness of the cornea was $49.2 \pm 2.2 \mu \mathrm{m}$. After BAC induction, epithelial cells of the eye cornea exhibited a poor arrangement, and the central thickness of the cornea dropped to $12.3 \pm 1.9 \mu \mathrm{m}$. Furthermore, some epithelial cell necrosis and stromal swelling were observed. T050SC produced better recovery than the control, and the central thickness of the cornea 
A
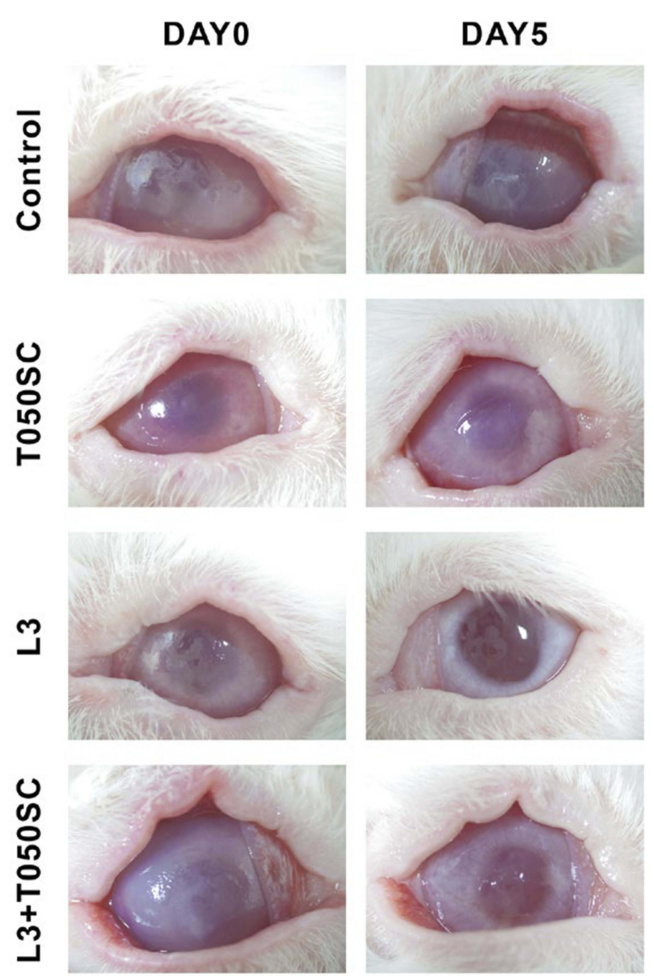

C Normal
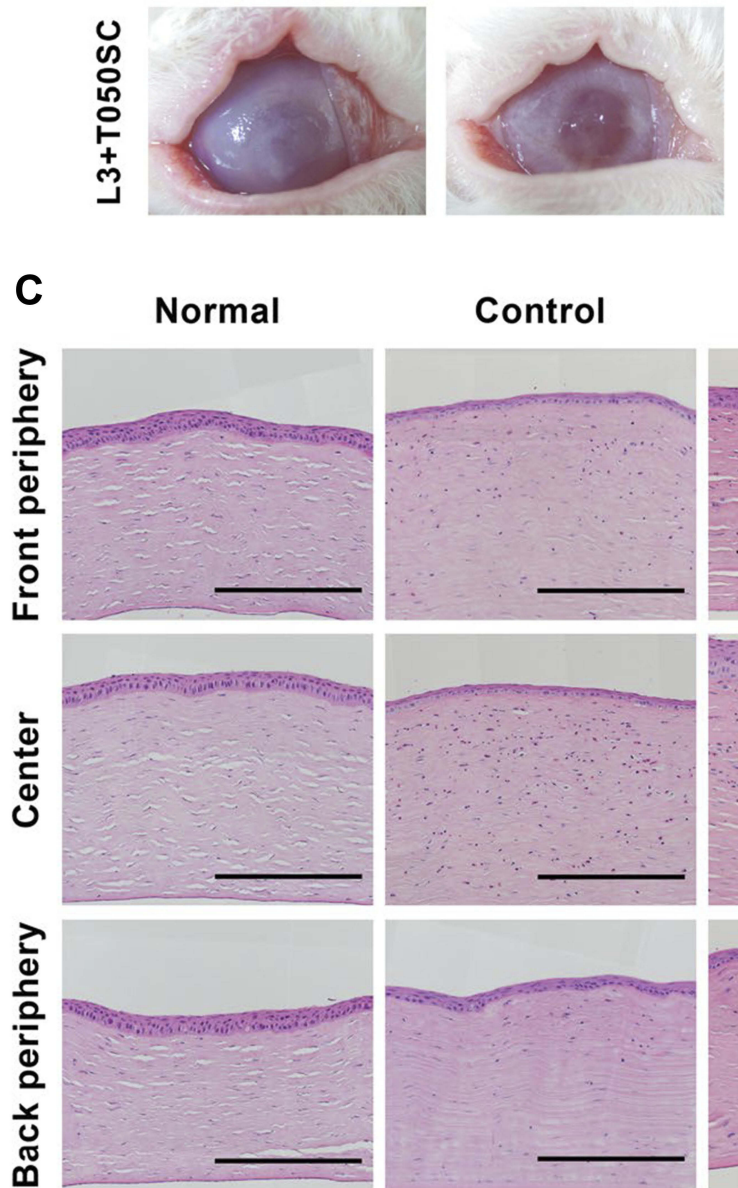

B

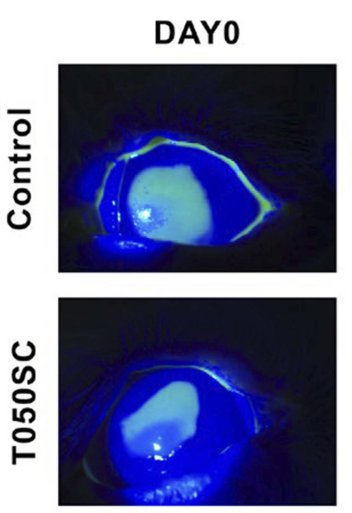

DAY5
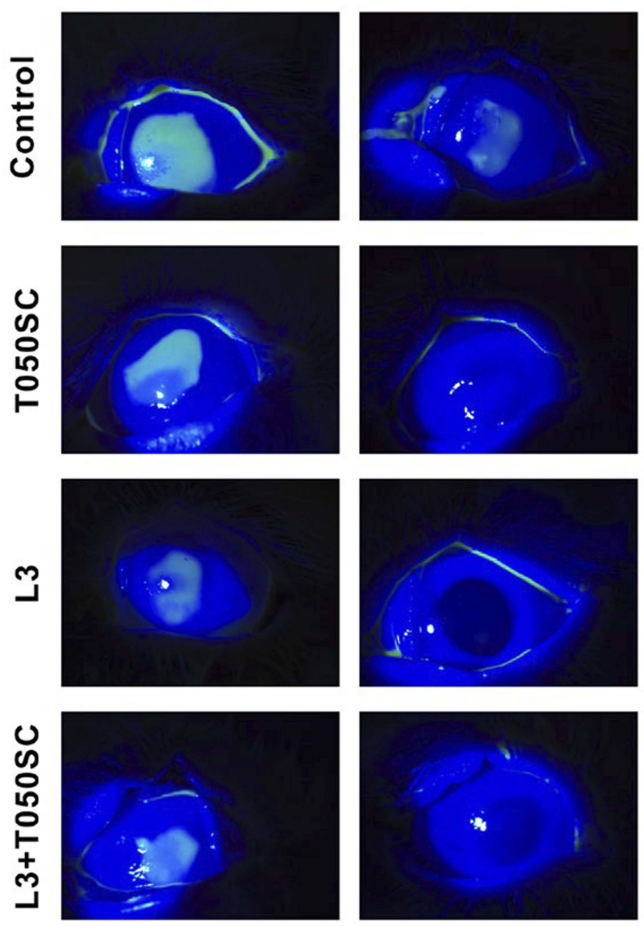

T050SC

L3

L3+T050SC
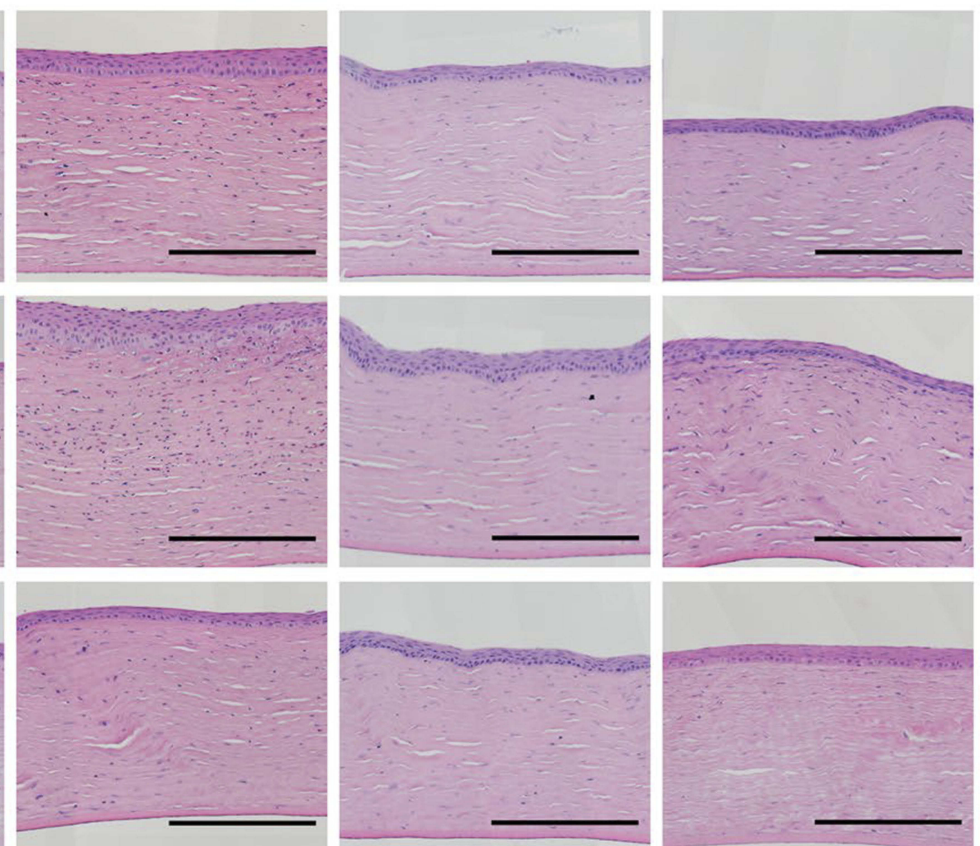

Figure 4 (A) Preoperative and postoperative appearances of the rabbit cornea. All groups presented a severe corneal ulceration appearance on day 0 . After 5 days of treatment with T033SC, L3, and L3+T050SC, photos were collected as postoperative appearances with a digital camera. (B) Eye corneas were stained with fluorescein, and photos were collected under cobalt-blue light ophthalmoscopy. (C) Histological analysis of normal rabbit cornea of untreated, and T050Sc-, L3-, and L3+T050SC-treated corneas on day 5 . Scale bars, $125 \mu \mathrm{m}$. 
Table I H\&E Staining of Corneal Severity Levels After 5 Days of Treatment

\begin{tabular}{|l|l|l|l|l|}
\hline Center Corneal Severity Level & Normal & Control & T050SC & L3 \\
\hline Corneal staining & None & Marked central & None & None \\
Center epithelial thickness $(n=2-4)$ & $49.2 \pm 2.2 \mu \mathrm{m}$ & $12.3 \pm 1.9 \mu \mathrm{m}$ & $45.1 \pm 1.2 \mu \mathrm{m}$ & $43.7 \pm 1.1 \mu \mathrm{m}$ \\
Epithelial damage & None & Severe & Moderate \\
Stromal histologic changes & None & Severe & Moderate & Slight \\
Endothelial histologic changes & None & None & None & Noderate \\
\hline
\end{tabular}

was $45.1 \pm 1.2 \mu \mathrm{m}$, which was similar to the thickness of the normal cornea. The arrangement of epithelial cells became more orderly, and no stromal swelling was observed. However, some eosinophil cell infiltration was observed, and Bowmen's layer became swollen, which may have been linked to the reason that the T050SC group had poor corneal transparency. The L3 and L3+T050SC groups showed promising corneal recovery after 5 days of treatment. Epithelial cells were well arranged, and the central thicknesses of the corneas were $43.7 \pm 1.1$ and $40.3 \pm 2.3 \mu \mathrm{m}$, respectively. However, the L3+T050SC group appeared to still be undergoing remodeling, as there were more fibroblast cells present in the upper part of the stroma and epithelial layer. The results indicated that T050SC and the growth factors secreted by PRP can repair severe DES of the cornea. However, L3 showed the best overall outcome. This may have resulted from the rapid release of growth factors, which play important roles in the early stage of healing of severe DES, whereas L3+T050SC instead exhibited sustained release. However, T050SC can act as a water-retention agent that provides a moist environment for DES and may result in better outcomes in the late stage of the healing process.

\section{Discussion}

SCNFs were prepared by chemical nanonization with TEMPO-oxidation in this study. The T050SC produced by chemical nanonization had a size of $539.2 \mathrm{~nm}$ with an $\mathrm{SD}$ of $49.35 \mathrm{~nm}$ and a zeta potential of $-51.6 \mathrm{mV}$. The vial-inversion method showed that $2 \%$ of T050SC nanofibers could form hydrogel after initial hydration and so formed hydrogel liquefied in water only after $30 \mathrm{~min}$ stirring. Probably, due to the gel-forming character with a viscous 3D network after hydration, T050SC nanofibers offered sustain release of growth factors from activated PRP

In the in vitro study examining chronic epithelial wound-healing regeneration to relieve DES, nanofibers of T050SC acted as plant-based scaffolds to provide a sustained-release effect of growth factors from activated PRP (L3). The sustained release of such factors can prolong the effect of the formulation, which can decrease the frequency of drug administration, thereby promoting higher convenience and patient compliance. The combination of T050SC and L3 showed the greatest enhancement in cell proliferation and promotion of cell migration leading to $89 \%$ healing of the wound. In the in vivo study of applying L3+T050SC to severe DES, L3+T050SC showed a better outcome of corneal ulcer relief than L3 alone. In the histological evaluation of rabbit eye corneas, results indicated that T050SC and growth factors secreted by PRP could repair corneas with severe DES. However, L3 produced the best overall outcomes. This may have resulted from the rapid release of growth factors that play important roles in the early stage of healing of severe DES, while L3+T050SC exhibited sustained release instead. However, T050SC can act as a water-retention agent that provides a moist environment for DES and may result in better outcomes in the later stages of the healing process.

\section{Conclusions}

TEMPO-oxidized SCNFs possessing a gel-forming character with a 3D network were developed as a functional scaffold with ideal characteristics. PRP combined with TEMPO-oxidized SCNFs designated as T050SC has pronounced potential applications in severe dry eye syndrome. These SCNFs combined with activated PRP also possesses conceivable medical practices for any epithelial cell disorders similar to severe dry eye syndrome. Furthermore, the multifunctional biomaterial based on SCNFs combined with activated PRP might have additional clinical applications such as hair regeneration in the future. However, the most challenge issue in practical applications of SCNFs combined with activated PRP is the stability of released various growth factors in the hydrogel. 


\section{Acknowledgments}

Taipei Medical University Hospital (107TMU-TMUH -13) and Ministry of Science and Technology, ROC (107-2314-B-038-035-MY3; 106-232-B-038-014-MY3) provided financial support is highly appreciated.

\section{Disclosure}

The authors report no conflicts of interest in this work.

\section{References}

1. Su CH, Sun CS, Juan SW, Hu CH, Ket WT, Sheu MT. Fungal mycelia as the source of chitin and polysaccharides and their applications as skin substitutes. Biomaterials. 1997;18(17):1169-1174.

2. Hung WS, Fang CL, Su CH, Lai WF, Chang YC, Tsai YH. Cytotoxicity and immunogenicity of SACCHACHITIN and its mechanism of action on skin wound healing. J Biomed Mater Res. 2001;56(1):93-100. doi:10.1002/1097-4636(200107)56:1<93::AIDJBM1072>3.0.CO;2-B

3. Su CH, Liu SH, Yu SY, et al. Development of fungal mycelia as a skin substitute: characterization of keratinocyte proliferation and matrix metalloproteinase expression during improvement in the wound-healing process. J Biomed Mater Res A. 2005;72(2):220-227. doi:10.1002/ jbm.a.30235

4. Chen RN, Lee LW, Chen LC, et al. Wound-healing effect of micronized sacchachitin (mSC) nanogel on corneal epithelium. Int J Nanomedicine. 2012;7:4697-4706. doi:10.2147/IJN.S34530

5. Chao FC, Wu MH, Chen LC, et al. Preparation and characterization of chemically TEMPO-oxidized and mechanically disintegrated sacchachitin nanofibers (SCNF) for enhanced diabetic wound healing. Carbohydrate Polymers. 2020;229:115507. doi:10.1016/j.carbpol.20 19.115507
6. Karim Z, Afrin S. Nanocellulose as novel supportive functional material for growth and development of cells. Cell Dev Biol. 2015;4:2. doi:10.4172/2168-9296.1000154

7. Alio JL, Arnalich-montiel F, Rodriguez AE. The role of "eye platelet rich plasma" (E-PRP) for wound healing in ophthalmology. Curr Pharm Biotechnol. 2012;13:1257-1265. doi:10.2174/1389201128006 24355

8. Avila MY, Igua AM, Mora AM. Randomised, prospective clinical trial of platelet-rich plasma injection in the management of severe dry eye. Br J Ophthalmol. 2018;1-6. doi:10.1136/bjophthalmol-2018312072

9. Alio JL, Rodriguez AE, WrbelDudzińska D. Eye platelet-rich plasma in the treatment of ocular surface disorders. Curr Opin Ophthalmol. 2015;26:325-332. doi:10.1097/ICU.0000000000000169

10. Ronci C, Ferraro AS, Lanti A, et al. Platelet-rich plasma as treatment for persistent ocular epithelial defects. Transfus Apher Sci. 2015; 52:300-304. doi:10.1016/j.transci.2014.12.027

11. Koulikovska M, Szymanowski O, Lagali N, et al. Platelet-rich plasma prolongs myofibroblast accumulation in corneal stroma with incisional wound. Curr Eye Res. 2015;40:1102-1110. doi:10.3109/ 02713683.2014.978478

12. Konstantinidis A, Hero M, Nanos P, Panos GD. Efficacy of autologous platelets in macular hole surgery. Clin Ophthalmol. 2013;7:745-750. doi:10.2147/OPTH.S44440

13. Engelmann K, Sievert U, Hölig K, et al. [Effect of autologous platelet concentrates on the anatomical and functional outcome of late stage macular hole surgery: a retrospective analysis]. Bundesgesundheitsblatt Gesundheitsforschung Gesundheitsschutz. 2015;58(11-12):1289-1298. doi:10.1007/s00103-015-2251-1. German.

14. Tseng CL, Seghatchian J, Burnouf T. Animal models to assess the therapeutic efficacy of human serum and serum-converted platelet lysates for dry eye syndrome: seeing is believing. Transfus Apher Sci. 2015;53(1):95-98. doi:10.1016/j.transci.2015.05.016
International Journal of Nanomedicine

\section{Publish your work in this journal}

The International Journal of Nanomedicine is an international, peerreviewed journal focusing on the application of nanotechnology in diagnostics, therapeutics, and drug delivery systems throughout the biomedical field. This journal is indexed on PubMed Central, MedLine, CAS, SciSearch ${ }^{\circledR}$, Current Contents ${ }^{\circledR} /$ Clinical Medicine,
Journal Citation Reports/Science Edition, EMBase, Scopus and the Elsevier Bibliographic databases. The manuscript management system is completely online and includes a very quick and fair peer-review system, which is all easy to use. Visit http://www.dovepress.com/ testimonials.php to read real quotes from published authors. 